\title{
Benefit with re-challenge of immune checkpoint inhibitors in patient with metastatic triple-negative breast cancer: a case report
}

\author{
Jinmei Zhou ${ }^{1}$, Tao Wang ${ }^{1}$, Li Bian ${ }^{1}$, Jianbin Li $^{2}$, Zefei Jiang ${ }^{1}$ \\ ${ }^{1}$ Breast Cancer Department of the Fifth Medical Center of Chinese PLA General Hospital, Beijing, China; ${ }^{2}$ Beijing Institute of Biotechnology, \\ Beijing, China \\ Correspondence to: Tao Wang. Breast Cancer Department of the Fifth Medical Center of Chinese PLA General Hospital, No. 8 Dongdajie, Beijing \\ 100071, China. Email: wangtao6@hotmail.com.
}

\begin{abstract}
Immune checkpoint inhibitors (ICIs) have shown promising efficacy in metastatic triple-negative breast cancer (TNBC), but there's no evidence on rechallenging with ICIs among patients who previously discontinued ICIs for reasons other than disease progression at the time of progression. We report a case of a 41-year-old female with metastatic TNBC who achieved significant disease reduction again through retreatment of ICIs at the time of progression after stopping ICIs which previously brought remarkable clinical benefit without confirmed progression. After failure of first-line rescue treatment including docetaxel and capecitabine, this patient enrolled an open-label, single center, phase I, dose escalation clinical trial which evaluated the safety, tolerability, and antitumor activity of JS001 in patients with advanced TNBC. Then she received ICI JS001-toripalimab and achieved partial response (PR) which was ongoing when she discontinued treatment for coronavirus disease 2019 (COVID-19) outbreak after 30 months' treatment duration. Eight months later, she rechallenged with toripalimab in combination with nab-paclitaxel when being confirmed disease progression and gained PR again after two cycles without serious adverse events (AEs). This case suggests that patients who experience disease progression after discontinuing ICIs for reasons other than disease progression could be able to rechallenge with ICIs and might achieve disease control.
\end{abstract}

Keywords: Breast cancer; triple-negative; immune checkpoint inhibitors (ICIs); rechallenge; case report

Received: 28 December 2020; Accepted: 16 March 2021; Published: 30 April 2021.

doi: $10.21037 /$ tbcr-20-71

View this article at: http://dx.doi.org/10.21037/tbcr-20-71

\section{Introduction}

Triple-negative breast cancer (TNBC) constitutes 15-20\% of all breast cancers and chemotherapy remains the primary treatment excluding bevacizumab and PARP inhibitors for selected subset (1). Prognoses of metastatic TNBC remain poor with median overall survival of approximately 18 months or less (2). The treatment of inhibitors of programmed death 1 (PD-1) and programmed death ligand 1 (PD-L1) is a breakthrough in the treatment history. Two phase 3 studies, Impassion 130 and KEYNOTE-355 have respectively demonstrated that atezolizumab or pembrolizumab in combination with chemotherapy can prolong median progression-free survival (PFS) in the PD-L1-positive patients. Furthermore, atezolizumab plus nab-paclitaxel showed a clinically meaningful increase of 7 months in median overall survival although without statistical significance $(3,4)$. In clinical practice, some patients who get benefits from immune checkpoint inhibitors (ICIs) may stop treatment for reasons other than disease progression or dose limiting toxicities, there's no evidence on rechallenging with ICIs among them at the time of progression yet. Here, we report a 41-yearold female with metastatic TNBC who achieved partial response (PR) again through rechallenge of ICIs at the time 

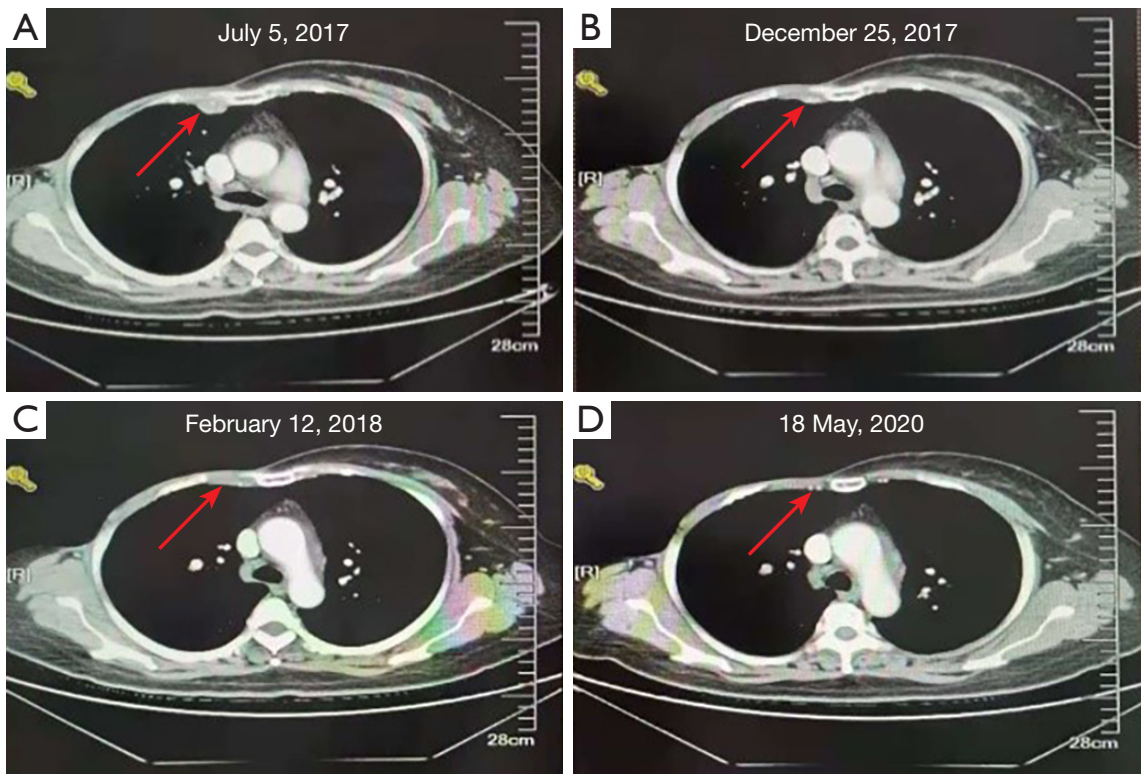

Figure $1 \mathrm{CT}$ scan show the pleural metastasis (red arrow) at pretreatment (A), 6 months after treatment (B), 8 months after treatment (C) and 4 months after treatment discontinuation (D).

of progression after discontinuing ICIs which previously brought remarkable clinical benefit without confirmed progression. We present the following article in accordance with the CARE reporting checklist (available at http:// dx.doi.org/10.21037/tbcr-20-71).

\section{Case presentation}

A 41-year-old female who was initially diagnosed with T2N1M0 TNBC was started on treatment with neoadjuvant chemotherapy including anthracycline and taxane with efficacy of stable disease (SD), modified radical mastectomy and adjuvant radiotherapy. Two years later, she was diagnosed with metastasis of bone and lymph node which was pathologically confirmed as TNBC. She received docetaxel plus capecitabine as first line treatment with efficacy of SD, the PFS was 6 months.

In July 2017, she was diagnosed with metastasis of bone, lymph node and pleura which was pathologically confirmed as TNBC again at our department. Then she enrolled one open-label, single center, phase I, dose escalation clinical trial which evaluated safety, tolerability, and antitumor activity of ICI-toripalimab in patients with locally advanced or metastatic TNBC. She achieved PR which was ongoing when she discontinued treatment without other regimens for coronavirus disease 2019 (COVID-19) outbreak after
30 months' treatment duration without serious adverse events (AEs) (Figure 1). Her PD-L1 expression was 70\% in pleura specimen [tumor proportion score (TPS), 22C3 antibody]. Her next generation sequencing by FOUNDATIONONE showed microsatellite statusstable, tumor mutational burden-low (4 Muts/Mb) and no mutations of therapeutic value. Eight months later, she was confirmed disease progression for appearance of swollen inguinal lymph node which was also pathologically confirmed as triple-negative. She rechallenged with toripalimab in combination with nab-paclitaxel and gained PR again after two cycles without serious AEs. Moreover, computed tomography (CT) of inguinal lymph showed a further reduction of the disease that confirmed PR after four cycles (Figure 2). All procedures performed in studies involving human participants were in accordance with the ethical standards of the institutional and/or national research committee(s) and with the Helsinki Declaration (as revised in 2013). The patient has given the consent for the case report to be published.

\section{Discussion}

The inhibition of PD-1/PD-L1 has been shown to have a good safety profile and clinical activity in patients with different types of tumors such as lung cancer, melanoma 

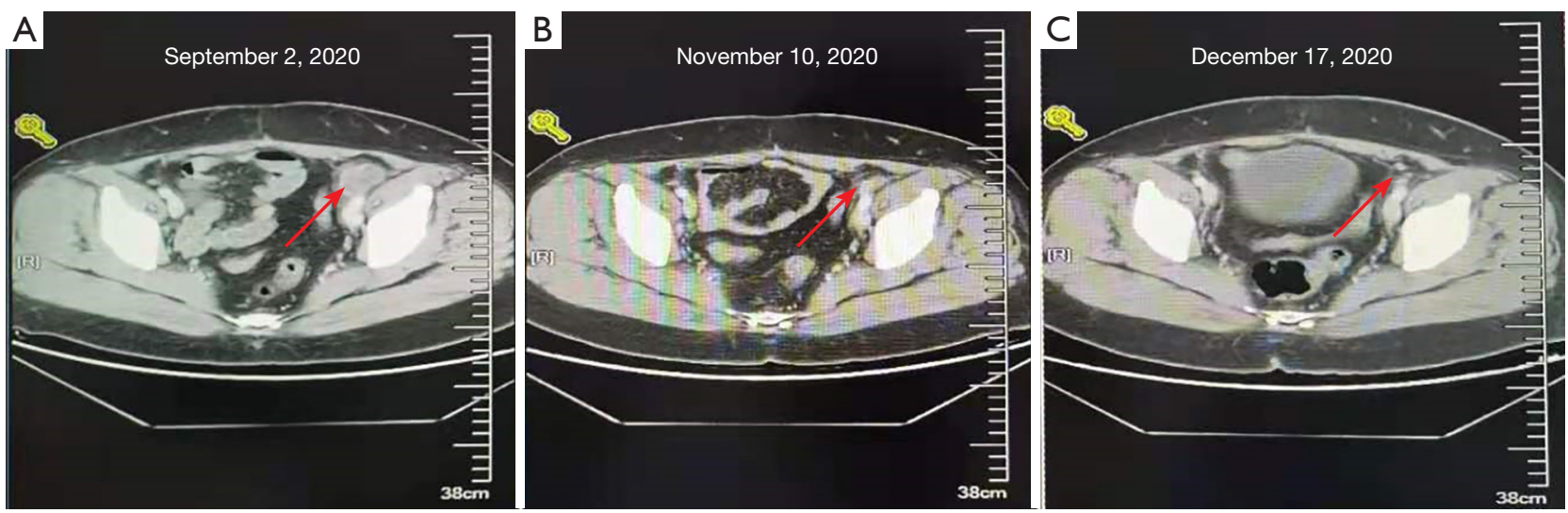

Figure 2 CT scan show the inguinal lymph node metastasis (red arrow) at pretreatment (A), two cycles after treatment (B), four cycles after treatment $(\mathrm{C})$.

and bladder cancer, which has significantly improved the prognosis and quality of life of patients and is the milestone progress in the treatment history. The ICIs as monotherapy or in combination with chemotherapy have also been investigated in breast cancer especially in TNBC which has higher prevalence of PD-L1 expression, higher tumor mutation burden and higher level of tumor infiltrating lymphocytes compared with hormone receptor-positive breast cancer subtypes. The activity of ICIs as monotherapy is relatively low with reported objective response rate (ORR) estimates of approximately $20 \%$ for first line and $5 \%$ or less for second line and more (5-8). The combination of chemotherapy and ICIs has successfully promoted the antitumor activity. IMpassion 130 showed the clinical benefit with atezolizumab plus nab-paclitaxel in patients with PD-L1 positive subset showing as ORR increasing from $42.6 \%$ to $58.9 \%$, PFS prolonging from 5.3 to 7.5 months and OS prolonging from 18 to 25 months. Based on these remarkable results, ICIs firstly got approvement for the treatment of breast cancer (4).

The majority of the most common ICIs related AEs are grade $1 / 2$, including fatigue, nausea, rash, fever and diarrhea, the rate of AEs of grade 3 or 4 is $10-15 \%$. The AEs of ICIs are different from chemotherapy's, but the AEs of special interest due to its unique mechanism such as immune-related hepatitis and so on need to be paid special attention (4-8). Patients in previous clinical trials of breast cancer received ICIs up to 2 years or until disease progression or unacceptable toxicity. The safety profile of ICIs was favorable, most AEs occurred within the first year of treatment and subsequent continuation didn't significantly increase toxicity which has been observed in clinical trials (8-10). The patient in our case report received ICIs up to 30 months with only grade1 AEs (11).

The most obvious difference between ICIs and chemotherapy or targeted therapy is the long-lasting and durable responses of ICIs, which means patients who benefit from ICIs can experience ongoing benefit for a long time after treatment discontinuation. In patients with melanoma or lung cancer for example, the duration of ICIs generally up to 2 years, but the activity persists after the end of treatment with the 2-year disease-free survival rate of $89.9 \%$ and $57.7 \%$ respectively $(9,12)$. The patient in our case report continued disease control up to 8 months after discontinuing treatment for COVID-19 outbreak. Some patients developed disease progression after ICIs discontinuation, there is a lack of evidence on the reinitiation of ICIs among them with only a few retrospective analyses $(10,13)$. The long-term outcome of the KEYNOTE-010 study of lung cancer showed that 32\% of patients who stopped treatment after completing 2 years treatment developed disease progression during follow-up, $56 \%$ of which received ICIs again and $78.6 \%$ got disease control $(9,10,13)$.

The optimal treatment opinion of our patient is platinum-containing regimen, chemotherapy in combination with bevacizumab or others? This patient achieved PR from toripalimab monotherapy with only grade1 AEs and the duration of response lasted for up to 38 months, including 8 months between treatment discontinuation and later disease progression. Based on the reasons described above and retrospective data of reinitiation of ICIs in other tumors, this patient could be able to rechallenge ICIs. In addition, one multicenter 
clinical trial-JS001-026-III-TNBC which evaluated toripalimab plus nab-paclitaxel compared with placebo plus nab-paclitaxel as a treatment for patients with unresectable locally advanced or metastatic TNBC was ongoing in our department. Although this patient did not meet the inclusion criteria because of previous use of toripalimab, we made one treatment strategy with reference to the clinical trial because it has initially shown good tolerability and efficacy. Finally, this patient received toripalimab plus nabpaclitaxel and achieved PR with no AEs.

\section{Conclusions}

Our experience suggests that patients with metastatic triple-negative breast cancer TNBC can be rechallenged with ICI based treatment regimens if their treatment was discontinued due to other reasons besides disease progression. Further larger studies may better elucidate the efficacy of ICI-based regimens in this setting.

\section{Acknowledgments}

Funding: This study was supported by Beijing Municipal Natural Science Foundation (7192198).

\section{Footnote}

Reporting Checklist: The authors have completed the CARE reporting checklist. Available at http://dx.doi.org/10.21037/ tbcr-20-71

Conflicts of Interest: All authors have completed the ICMJE uniform disclosure form (available at http://dx.doi. org/10.21037/tbcr-20-71). ZJ serves as an Editor-inChief of Translational Breast Cancer Research. JL serves as an unpaid Managing Editor of Translational Breast Cancer Research. The other authors have no conflicts of interest to declare.

Ethical Statement: The authors are accountable for all aspects of the work in ensuring that questions related to the accuracy or integrity of any part of the work are appropriately investigated and resolved. All procedures performed in studies involving human participants were in accordance with the ethical standards of the institutional and/or national research committee(s) and with the Helsinki Declaration (as revised in 2013). The patient has given the consent for the case report to be published.
Open Access Statement: This is an Open Access article distributed in accordance with the Creative Commons Attribution-NonCommercial-NoDerivs 4.0 International License (CC BY-NC-ND 4.0), which permits the noncommercial replication and distribution of the article with the strict proviso that no changes or edits are made and the original work is properly cited (including links to both the formal publication through the relevant DOI and the license). See: https://creativecommons.org/licenses/by-nc-nd/4.0/.

\section{References}

1. Foulkes WD, Smith IE, Reis-Filho JS. Triple-negative breast cancer. N Engl J Med 2010;363:1938-48.

2. Cardoso F, Senkus E, Costa A, et al. 4th ESO-ESMO International Consensus Guidelines for Advanced Breast Cancer (ABC 4)†. Ann Oncol 2018;29:1634-57.

3. de Melo Gagliato D, Buzaid AC, Perez-Garcia J, et al. Immunotherapy in breast cancer: current practice and clinical challenges. BioDrugs 2020;34:611-23.

4. Schmid P, Rugo HS, Adams S, et al. Atezolizumab plus nab-paclitaxel as first-line treatment for unresectable, locally advanced or metastatic triple-negative breast cancer (IMpassion130): updated efficacy results from a randomised, double-blind, placebo-controlled, phase 3 trial. Lancet Oncol 2020;21:44-59.

5. Nanda R, Chow LQ, Dees EC, et al. Pembrolizumab in patients with advanced triple-negative breast cancer: phase Ib KEYNOTE-012 study. J Clin Oncol 2016;34:2460-7.

6. Adams S, Loi S, Toppmeyer D, et al. Pembrolizumab monotherapy for previously untreated, PD-L1-positive, metastatic triple-negative breast cancer: cohort $\mathrm{B}$ of the phase II KEYNOTE-086 study. Ann Oncol 2019;30:405-11.

7. Adams S, Schmid P, Rugo HS, et al. Pembrolizumab monotherapy for previously treated metastatic triplenegative breast cancer: cohort A of the phase II KEYNOTE-086 study. Ann Oncol 2019;30:397-404.

8. Emens LA, Cruz C, Eder JP, et al. Long-term clinical outcomes and biomarker analyses of atezolizumab therapy for patients with metastatic triple-negative breast cancer: a phase 1 study. JAMA Oncol 2019;5:74-82.

9. Herbst RS, Garon EB, Kim DW, et al. Long-term outcomes and retreatment among patients with previously treated, programmed death-ligand 1-positive, advanced non-small-cell lung cancer in the KEYNOTE-010 study. J Clin Oncol 2020;38:1580-90.

10. Waterhouse DM, Garon EB, Chandler J, et al. Continuous versus 1 -year fixed-duration nivolumab in previously 
treated advanced non-small-cell lung cancer: CheckMate 153. J Clin Oncol 2020;38:3863-73.

11. Bian L, Zhang H, Wang T, et al. JS001, an anti-PD-1 mAb for advanced triple negative breast cancer patients after multi-line systemic therapy in a phase I trial. Ann Transl Med 2019;7:435.

12. Robert C, Ribas A, Hamid O, et al. Durable complete response after discontinuation of pembrolizumab in patients with metastatic melanoma. J Clin Oncol 2018;36:1668-74.

13. Tikkanen A, Iivanainen S, Koivunen JP. Treatment discontinuation and re-initiation of anti-PD-(L)1 agents in metastatic cancers. J Cancer Res Clin Oncol 2020;146:2153-60. doi: $10.21037 /$ tbcr-20-71

Cite this article as: Zhou J, Wang T, Bian L, Li J, Jiang Z. Benefit with re-challenge of immune checkpoint inhibitors in patient with metastatic triple-negative breast cancer: a case report. Transl Breast Cancer Res 2021;2:14. 